\title{
Paper
}

UDC $621.039 .341: 513.012: 681.3 .02$

\section{An Analytical Solution of the Neutron Diffusion Equation in Toroidal Coordinates}

\author{
By \\ Masaharu Seki Ikuo Kaji \\ Member Member
}

\begin{abstract}
Summary
Analytical solutions of the neutron diffusion equation in the non-separable toroidal coordinate system are useful for analyses of diffusion phenomena described in the toroidal coordinate, though the solutions have not been obtained yet. By using the method developed by Weston, the problem of solving the partial differential equation in the three variables in which only one variable is separable is reduced to solving a recurrent set of ordinary differential equations in one variable. Thus, analytical solutions are obtained for the neutron diffusion equation in the toroidal coordinate. These solutions form a complet set of the solutions, satisfying the condition for representing the diffusion from a point source when the fundamental length in the toroidal coordinate $d$ approaches zero.

As an example of applications of the solutions, the monoenergy neutron flux which takes a given value around the axisymmetrical toroidal neutron source is evaluated with high accuracy, without using the approximation of the expansion with respect to the inverse aspect ratio.
\end{abstract}

\section{Introduction}

Currently, one of the promising concepts for achieving controlled themonuclear fusion reactor is the Tokamak system whose geometry is basically toroidal. In studing possible reactor designs, neutronic and photonic calculations play very important role. G. C. Pomraning and C. A. Stevens $s^{(1)}$ have showed that for an azimuthally independent problem, the toroidal diffusion equation can be cast into the standard $r-\theta$ cylindrical equation. An idealized problem has been solved analytically to obtain the first-order correction term from overall curvature of toroidal system. J. Jung ${ }^{(2)}$ has presented a numerical method for solv* ing the transport equation in toroidal geometry, which can be implemented in an existing two-dimensional $S_{N}$ code.

We present an analytical method for solving the monoenergitic neutron diffusion equation in toroidal coordinates and show an example of computation of

Masaharu Seki is with Department of Industrial Enginnering. Hokkaido Automotive Junior College \& Ikuo Kajt is with Faculty of Engineering, Hokkaido University. Manuscript received Mar. $22,1983$. the solution. The neutron diffusion equation $\nabla^{2} \Psi$ $-k^{2} \Psi=0^{(3)}$ is non-separable for the toroidal coordinate as for the Helmholtz equation. The equation in three variables can be separated to the ordinary differential equation in one variable and the partial differential equation in two non-separable variables in the toroidal coordinate. A method has been developed by Weston ${ }^{(4)(5)}$ for solving the Helmholtz equation for a class of non-separable coordinate systems. With this method, the problem of solving the partial differential equation in two variables can be reduced to solving a recurrent set of ordinary differential equations in one variable. Thus, we can solve analytically the neutron diffusion equation for the exterior and interior problems in toroidal coordinates ${ }^{(6)}$, to describe diffusion phenomena from a ring source. The solutions are represented with the double infinite series of the associated Legendre functions $P_{\nu}^{\mu}(z)$ whose argument is the aspect ratio $z$ of a torus.

As an example of applications of the solutions, we evaluate the monoenergy neutron flux which takes a given value around the axisymmetrical toroidal neutron source $\left(z=z_{s}=R_{s} / r_{s}\right)$ in the toroidal coordinate and show the equiflux curves in figures.

本稿は Section E (Trans. I. E. E. of Japan, Vol. 104, No. 5/6, May/June, p. 85, 1984) がらの転載である。 


\section{The Analytical Solution of the Neutron Diffusion Equation}

We introduce the toroidal coordinate $(\sigma, \phi, \phi)^{(7) \sim(9)}$ as shown in Fig. 1, which is defined $\log (A P / B P)=\sigma$, $\angle A P B=\phi$ and azimuthal angle $\phi$ around $w$-axis. The metric coefficients of toroidal coordinates are given by

$$
\left.\begin{array}{l}
h_{1}=h_{2}=d / g \\
h_{3}=d \sin \sigma / g
\end{array}\right\}
$$

where $g=\cosh \sigma-\cos \phi$. For fixed values of $\sigma$ and $\phi$, the argument $\phi$ describes the Apollonius circle, so that the fundamental length $d$ is given by $d^{2}=R^{2}-r^{2}$ where $R$ and $r$ are the major and minor radii of a torus, respectively. On the Apollonius circle, we obtain

$$
\cosh \sigma=R / r, \quad \sinh \sigma=d / r,
$$

where $z=R / r$ is an aspect ratio.

The neutron diffusion equation

$$
\nabla^{2} \Psi-k^{2} \Psi=0
$$

is expressed as

$$
\begin{aligned}
& \frac{1}{h_{1} h_{2} h_{3}}\left\{\frac{\partial}{\partial \sigma}\left(h_{3} \frac{\partial \Psi}{\partial \sigma}\right)+\frac{\partial}{\partial \psi}\left(h_{3} \frac{\partial \Psi}{\partial \psi}\right)\right. \\
& \left.+\frac{h_{1} h_{2}}{h_{3}} \frac{\partial^{2} \Psi}{\partial \phi^{2}}\right\}-k^{2} \Psi=0
\end{aligned}
$$

in the toroidal coordinates. The basic solutions of Eq. (4) are represented by

$$
\begin{aligned}
& \widehat{\Psi}_{e T}^{m}(z, \phi, \phi)=e^{i m \phi} \sum_{\nu=T}^{\infty} A_{\nu}(z) g^{-\nu}, \\
& \widehat{\Psi}_{o T^{\prime}}^{m}(z, \phi, \phi)=e^{i m \phi} \sin \phi \sum_{\nu=T^{\prime}}^{\infty} B_{\nu}(z) g^{-\nu},
\end{aligned}
$$

where $\widehat{\Psi}_{e T}^{m}$ and $\widehat{\Psi}_{o r}^{m}$ are the even and odd functions, respectively. Substituting Eqs. ( 5 ) and ( 6 ) into Eq. (4), we obtain the recurrent sets of ordinary differential equations with infinite unknowns $A_{\nu}$ and $B_{\nu}$ :

$$
\left.\begin{array}{c}
\left(z^{2}-1\right) A_{\nu}^{\prime \prime}+2 z A_{\nu}^{\prime}-A_{\nu}\{\nu(\nu+1) \\
\left.+m^{2} /\left(z^{2}-1\right)\right\}+F_{\nu}(z)=0 \\
F_{\nu}(z)=-k^{2} d^{2} A_{\nu-2} \\
-(2 \nu-1)\left\{\left(z^{2}-1\right) A_{\nu-1}^{\prime}\right. \\
\left.-z(\nu-1) A_{\nu-1}\right\} \\
(\nu=T, T+1, T+2, \cdots)
\end{array}\right\}
$$

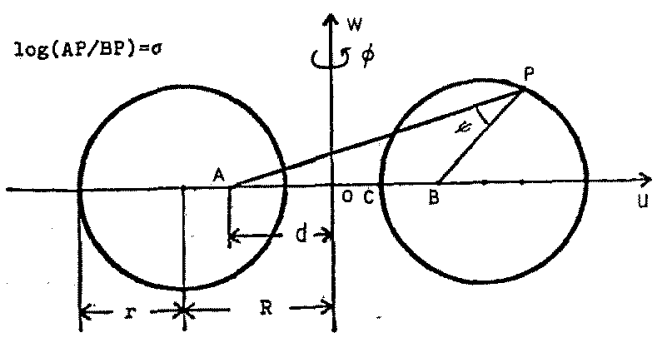

$d^{2}=R^{2}-r^{2}, \cosh \sigma=R / r$ (aspect ratio)

Fig. 1. Toroidal coordinates $(\sigma, \phi, \phi)$.

$$
\left.\begin{array}{c}
\left(z^{2}-1\right) B_{\nu}^{\prime \prime}+2 z B_{\nu}^{\prime}-B_{\nu}\{\nu(\nu-1) \\
\left.+m^{2} /\left(z^{2}-1\right)\right\}+G_{\nu}(z)=0 \\
G_{\nu}(z)=-k^{2} d^{2} B_{\nu-2} \\
-(2 \nu-1)\left\{\left(z^{2}-1\right) B_{\nu-1}^{\prime}\right. \\
\left.-z(\nu-2) B_{\nu-1}\right\} \\
\left(\nu=T^{\prime}, T^{\prime}+1, T^{\prime}+2, \cdots\right)
\end{array}\right\},
$$

where the prime denotes differentiation with respect to $z$. The numbers $T$ and $T^{\prime}$ are determined from the boundary conditions. Since $F_{\nu}(z)=0$ and $G_{\nu}(z)=0$ for $\nu=T$ and $\nu=T^{\prime}$, respectively, i. e. $A_{\nu}=0$ and $B_{\nu}=$ 0 for $\nu<T$ and $\nu<T^{\prime}$, Eqs. (7) and (8) or $\nu=T$ and $T^{\prime}$ become homegeneous equations whose solutions are the associated Legendre functions $P_{\nu}^{m}(z)$ and $Q_{\nu}{ }^{m}(z)^{(7) \sim(9)}$. Putting $\omega_{\nu}^{m}(z)=P_{\nu}^{m}(z)$ for $Q_{\nu}^{m}(z)$, solutions or Eqs. (7) and ( 8 ) are given respectively by

$$
\begin{aligned}
A_{\nu}(z)= & \omega_{\nu}^{m}(z) \int_{c_{1}}^{z} \frac{d y}{\left(1-y^{2}\right)\left[\omega_{\nu}^{m}(y)\right]^{2}} \\
& \times \int_{c_{z}}^{y} F_{\nu}(x) \omega_{\nu}^{m}(x) d x, \\
B_{\nu}(z)= & \omega_{\nu-1}^{m}(z) \int_{c_{1}}^{z} \frac{d y}{\left(1-y^{2}\right)\left[\omega_{\nu-1}^{m}(y)\right]^{2}} \\
& \times \int_{c_{2}^{\prime}}^{y} G_{\nu}(x) \omega_{\nu-1}^{m}(x) d x,
\end{aligned}
$$

where the constants $c_{1}, c_{2}, c_{1}^{\prime}$ and $c_{2}^{\prime}$ are determined from the boundary conditions. The solution of Eq. (4) can be represented by the linear combination of the basic solutions $\widehat{\Psi}_{e p}^{m}$ and $\widehat{\Psi}_{o p^{\prime}}^{m}$ in Eqs. (5) and (6) where $A_{p}(z)$ and $B_{p}{ }^{\prime}(z)$ are equal to $\omega_{p}^{m}(z)$ and $\omega_{p}^{m}-1$ $(z)$, respectively, and the constants $c_{1}, c_{2}, c_{1}^{\prime}$ and $c_{2}{ }^{\prime}$ are all set to unity, where $p=T, T+, T+2, \cdots$ and $p^{\prime}=T^{\prime}, T^{\prime}+1, T^{\prime}+2, \cdots$. Since we shall be interested in solutions periodic in the angle $\phi$, we set $m=0, \pm 1$, $\pm 2, \cdots$. For convenience the symbol $M$ will be used to signify $|m|$, i. e. $M=|m|$. By defining the integral operator $I(M, \nu)$ operating on the function $F(z)$ as follows:

$$
\begin{aligned}
I(M, \nu) F(z)= & \omega_{\nu}^{M}(z) \int_{1}^{z} \frac{d y}{\left(1-y^{2}\right)\left[\omega_{\nu}^{M}(y)\right]^{2}} \\
& \times \int_{1}^{y} \omega_{\nu}^{M}(x) F(x) d x, \quad \text { (11) }
\end{aligned}
$$

Eqs. (9) and (10) with $c_{1}=c_{2}=c_{1}{ }^{\prime}=c_{2}^{\prime}=1$ is rewritten respectively as

$$
\begin{aligned}
& A_{\nu}(z)=I(M, \nu) F_{\nu}(z) \\
& B_{\nu}(z)=I(M, \nu-1) G_{\nu}(z) .
\end{aligned}
$$

To calculate $A_{\nu}(z)$ and $B_{\downarrow}(z)$ from Eqs. (12) and (13), we use the following lemma which has been presented by Weston:

[Lemma] If for $X>0$,

$$
\begin{aligned}
& G(M, X, X-\Lambda)=\left(z^{2}-1\right)^{X / 2} P_{X-\Lambda-1}^{-M-X}(z), \\
& F(z)= a\left\{\left(z^{2}-1\right) \frac{d}{d z} G(M, X-1, X-\Lambda)\right. \\
&-z(\Lambda-1) G(M, X-1, X-\Lambda)\} \\
&+b G(M, X-1, X-\Lambda+1),
\end{aligned}
$$


then

$$
\begin{aligned}
I(M, \Lambda) F(z)= & -\{a(M+2 X-\Lambda-1)+b\} \\
& \times G(M, X, X-\Lambda) /(2 X) .
\end{aligned}
$$

Now, we integrate the right hand sides of Eqs. (9) and (10) by the use of the lemma and construct solutions of the neutron diffusion equation.

\subsection{General solutions of the neutron diffusion equation in toroidal coordinates}

We find appropriate solutions for solving diffusion phenomena described in the toroidal coordinates $(\sigma$, $\psi, \phi)$. By the use of the lemma, we obtain the coef. ficients $A_{\nu}(z)$ and $B_{\nu}(z)$ from Eqs. ( 9$)$ and (10), respectively, and find the solutions $\bar{\Psi}_{e p}^{m}$ and $\bar{\Psi}_{o p}^{m}$, from Eqs. ( 5 ) and (6) which are expressed as the series of the $A_{\nu}(z)$ and $B_{\nu}(z)$, respectively. Therefore, we obtain four linear combinations of the solutions $\bar{\psi}_{e p}^{m}$ and $\hat{\Psi}_{o p}^{m}$, which form a complet set of the solutions of Eq. (4), describing diffusion phenomena due to a ring source ${ }^{(5)}$, as follows :

$$
\begin{aligned}
& \Phi_{e N}^{m}=(-1)^{M}\left(S_{-N-1}^{M}-S_{N}^{M}\right) e^{i m \phi}, \\
& \Psi_{e N}^{m}=S_{N}^{N} e^{i m \phi} \text {, } \\
& \Phi_{o N^{\prime}}^{m}=(-1)^{M+1}\left(T_{-N^{\prime}-1}^{M}-T_{N^{M}}^{M}\right) e^{i m \phi}, \\
& \Psi_{o N^{\prime}}^{m}=T_{N^{\prime}}^{M} e^{i m \phi} \text {, } \\
& S_{L}^{M}=\frac{\Gamma(L+M+1) \pi^{1 / 2}(k d)^{L}(-1)^{M}}{\Gamma(L-M+1) 2^{(M+L) / 2+1} g^{(M+L) / 2}} \\
& \times \sum_{p=0}^{\infty} \frac{(k d)^{2 p} K_{p}^{1}}{2^{p} g^{p}} \\
& K_{p}^{1}=\frac{1}{p ! \Gamma(L+3 / 2+p)} \\
& \times \sum_{q=0}^{l} \frac{\left(\frac{M-L}{2}\right)_{q}\left(\frac{L+M+1}{2}\right)_{q}\left(z^{2}-1\right)^{q / 2}}{q ! g^{q}} \\
& \times P_{p+(L+M) / 2}^{-M-q}(z) \text {, } \\
& (\gamma)_{q}=\gamma(\gamma+1)(\gamma+2) \cdots(\gamma+q-1) \\
& (\gamma)_{0}=1 \\
& M=|m|=0,1,2,3, \cdots \\
& L=N \text { or }-N-1 \\
& N=M+2 l, l: \text { positive integer or zero } \\
& g=z-\cos \psi, z=\cosh \sigma
\end{aligned}
$$

$$
\begin{aligned}
T_{L}^{M}= & \frac{\Gamma(L+M+1) \pi^{1 / 2}(k d)^{L}(-1)^{M} \sin \psi}{\Gamma(L-M+1) 2^{(M+L+1) / 2} g^{(M+L+1) / 2}} \\
& \times \sum_{p=0}^{\infty} \frac{(k d)^{2 p} K_{p}^{2}}{2^{p} g^{p}} \\
K_{p}^{2}= & \frac{1}{p ! \Gamma(L+3 / 2+p)} \\
\times & \sum_{q=0}^{l} \frac{\left(\frac{M-L+1}{2}\right)_{q}\left(\frac{M+L}{2}+1\right)_{q}\left(z^{2}-1\right)^{q / 2}}{q ! g^{q}} \\
& \times P_{p+(M-q+L-1) / 2}^{-M}(z), \\
L= & N^{\prime} \text { or }-N^{\prime}-1 \\
N^{\prime} & =M+2 l+1, l: \text { positive integer or zero }
\end{aligned}
$$

where $\Gamma$ is the gamma function, and $S_{L}^{M}$ and $T_{L}^{M}$ are respectively even and odd functions for $\psi$. Experssions (17). (19) and (18), (20) represent solutions of the diffusion equation ( 3 ) for the exterior and interior problems of a torus with a ring source at $z=z_{s}$ respectively.

\subsection{The condition representing the diffusion from a ring source}

We have found the linear combinations $(17) \sim(20)$ representing solutions of the diffusion equation, describing diffusion phenomena due to a ring source in subsection 2.1. We shall prove here that the solutions satisfy the following necessary condition for representing the diffusion from a point source when $d$ approaches zero:

$$
\begin{aligned}
& \lim _{d \rightarrow 0} \Phi_{e N}^{m} \text { and } \lim _{d \rightarrow 0} \Phi_{o N}^{m} \\
& \quad=\sqrt{\frac{2}{\pi k R}} K_{N+1 / 2}(k R) P_{N}^{M}(\cos \theta) e^{i m \phi}, \\
& \lim _{d \rightarrow 0} \Psi_{e N}^{m} \text { and } \lim _{d \rightarrow 0} \Psi_{o N}^{m} \\
& =\sqrt{\frac{\pi}{2 k R}} I_{N+1 / 2}(k R) P_{N}^{M}(\cos \theta) e^{i m \phi},
\end{aligned}
$$

where $(R, \theta, \phi)$ are spherical polar coordinates, $K_{N+1 / 2}$ and $I_{N+1 / 2}$ are the modified Bessel functions and $P_{N}^{M}$ is the associated Legendre function.

[Proof] The associated Legendre function is expressed as

$$
\begin{aligned}
P_{\nu}^{-m}(\cos \theta)= & \frac{(\sin \theta)^{m}}{m ! 2 m} F\left(\frac{m+\nu+1}{2},\right. \\
& \left.\frac{m-\nu}{2},|m+1|, \sin ^{2} \theta\right),
\end{aligned}
$$

in spherical polar coordinates, where $F$ is the Gauss' hypergeometic function. When $d$.approaches zero, the relation between the toroidal and spherical polar coor. dinates is given by

$$
\begin{aligned}
& \left(z^{2}-1\right)^{1 / 2} \sim 2(d / R) \sin \theta \\
& (z-\cos \psi) \sim 2(d / R)^{2} \\
& z \sim 1+2(d / R)^{2} \sin ^{2} \theta \\
& \sin \psi \sim 2(d / R) \cos \theta
\end{aligned}
$$

Therefore, we obtain when $d$ approaches zero

$$
P_{P+(L+M): 2}^{-M-q}(z) \sim \frac{(d / R \cdot 2 \sin \theta)^{M+q}}{(M+q) ! 2^{M+q}},
$$

from Eqs. (27) and (28). Substituting Eq. (29) into Eq. (22), we have

$$
\begin{aligned}
K_{p}^{1} \sim & \frac{(d / R \sin \theta)^{M}}{p ! \Gamma(L+3 / 2+p)} \\
& \times \sum_{q=0}^{l} \frac{\left(\frac{M-L}{2}\right)_{q}\left(\frac{L+M+1}{2}\right)_{q}}{q !(M+q) !}\left(\sin ^{2} \theta\right)^{q} .
\end{aligned}
$$

Using Eq. (30) and the expressions of the modified Bessel function and associated Legendre function, the 
asymptotic form of Eq. (21) is obtained for $d$ approaching zero, as follows:

$$
\begin{aligned}
\lim _{d \rightarrow 0} S_{L}^{M}= & \frac{\Gamma(L+M+1) \pi^{1 / 2}(-1)^{M}(k R / 2)^{L}}{\Gamma(L-M+1) \cdot 2} \\
& \times \sum_{p=0}^{\infty} \frac{(k R / 2)^{2 p}}{p ! \Gamma(L+3 / 2+p)} \frac{(\sin \theta)^{M}}{2^{M} \Gamma(M+1)} \\
& \times F\left(\frac{L+M+1}{2}, \frac{M-L}{2}, M+1, \sin ^{2} \theta\right) \\
= & (-1)^{M} \frac{\Gamma(L+M+1)}{\Gamma(L-M+1)} \frac{\pi^{1 / 2}(k R / 2)^{L}}{2} \\
& \times \sum_{p=0}^{\infty} \frac{(k R / 2)^{2 p}}{p ! \Gamma(L+3 / 2+p)} P_{N}^{M}(\cos \theta) \\
= & \frac{\sqrt{\pi}}{2} \sqrt{\frac{2}{k R} \sum_{p=0}^{\infty} \frac{(k R / 2)^{2 p+L+1 / 2}}{p ! \Gamma(L+3 / 2+p)}} \\
& \times P_{L}^{M}(\cos \theta) \\
= & \sqrt{\frac{\pi}{2 k R}} I_{L+1 / 2}(k R) P_{L}^{M}(\cos \theta) .
\end{aligned}
$$

In a manner similar to the above, we get

$$
\begin{aligned}
& \lim _{d \rightarrow 0}(-1)^{M}\left(S_{-N-1}^{M}-S_{N}^{M}\right) \\
& =\sqrt{\frac{2}{\pi k R}} K_{N+1 / 2}(k R) P_{N}^{M}(\cos \theta) \\
& \lim _{\alpha \rightarrow 0}(-1)^{M+1}\left(T_{-N^{\prime}-1}^{M}-T_{N^{M}}^{M}\right) \\
& =\sqrt{\frac{2}{\pi k R}} K_{N^{\prime}+1 / 2}(k R) P_{N}^{M}(\cos \theta) \\
& \lim _{d \rightarrow 0} T_{N^{\prime}}^{M}=\sqrt{\frac{\pi}{2 k R}} I_{N^{\prime}+1 / 2}(k R) P_{N^{\prime}}^{M}(\cos \theta)
\end{aligned}
$$

(Q. E. D.)

\subsection{The convergence of the solution of diffusion equation}

We considerce the convergence of expressions $S_{L}^{M}$ and $T_{L}^{M}$ given by Eqs. (21) and (23) respectively. The expressions comprise of two series, one finite and the other infinite. Hence for the convergence, we need to consider only the summation over $p$. To show convergence of the above series, we require the following inequality

$$
\begin{aligned}
P_{\nu}^{-M}(z) \leq & (z-1)^{M / 2}(z+1)^{M / 2} \\
& \times\left[z \pm\left(z^{2}-1\right)^{1 / 2}\right)^{\nu} / \Gamma(1+M),
\end{aligned}
$$

where the positive and negative signs are taken for $\nu$ $\geq 0$ and $\nu<0$, respectively. The infinite series of Eq.

(21) is evaluated as

$$
\begin{aligned}
S_{L}^{M} \leq & \frac{\Gamma(L+M+1) \pi^{1 / 2}(k d)^{L}}{\Gamma(L-M+1) 2^{(M+L) / 2+1} g^{(M+L) / 2}} \\
& \times\left(\frac{z-1}{z+1}\right)^{M}\left(z \pm \sqrt{z^{2}-1}\right)^{(L+M) / 2} \sum_{q=0}^{l}\left(\frac{z-1}{z+1}\right)^{q} \\
& \times \frac{\left(\frac{M-L}{2}\right)_{q}\left(\frac{L+M+1}{2}\right)_{q}\left(z^{2}-1\right)^{q / 2}}{\Gamma(M+q+1) q ! g^{q}} \sum_{p=0}^{\infty} u_{p},
\end{aligned}
$$

$$
u_{p}=\frac{(k d)^{2 p}\left(z+\sqrt{z^{2}-1}\right]^{p}}{2^{p} p ! \Gamma(L+3 / 2+p)(z-\cos \phi)^{p}} .
$$

We now obtain, when $p \gg 1$,

$$
\frac{u_{p}}{u_{p-1}} \sim \frac{(k d)^{2}}{p^{2}} \frac{z \pm \sqrt{z^{2}-1}}{z-\cos \psi}<1
$$

The condition (35) shows that the infinite series in Eq. (34) is convergent. For $z \gg 1, \mathrm{Eq}$. (34) becomes

$$
\begin{aligned}
S_{L}^{M} & \frac{\Gamma(L+M+1) \pi^{1 / 2}(k d)^{L}(-1)^{M}}{\Gamma(L-M+1) 2^{(M+L) / 2+1}} \\
& \times \frac{(2 z)^{|M+L / 2|}}{z^{(M+L) / 2}} \\
& \times \sum_{q=0}^{\iota} \frac{\left(\frac{M-L}{2}\right)}{\Gamma(M+q+1) q !} \frac{\left(\frac{L+M+1}{2}\right)_{q}}{\sum_{p=0}^{\infty}} u_{p} .
\end{aligned}
$$

Hence, the expression $S_{L}^{M}$ with $L=N=M+2 l$ has a finite value when $z$ approaches $\infty$. As the expression $S_{L}^{M}$ with $L=-N-1$ has an infinite value for $z$ approaching $\infty$, the expression $\Phi_{e^{N}}^{m}$ in Eq. (17) has a singularity at $z=\infty$. In subsection 2.2 , we have found that the expressions $S_{L}^{M}$ and $(-1)^{M}\left(S_{-N-1}^{M}-S_{N}^{M}\right)$ are represented by Eqs. (31) and (32) for $z=R / r$ approaching unity, i. e. $d$ approaching zero. Therefore, the expressions $\Phi_{e N}^{m}$ and $\Psi_{e N}^{m}$ can be adoped for the solutions of the diffusion equation for the exterior and interior problems, respectively. In a manner similar to the above, it is proved that the expressions $\phi_{\text {oN }}^{\text {nd }}$ in Eq. (19) and $\Psi_{O_{N}}^{m}$ in Eq. (20) have a singularity and a finite value at $z=\infty$, respectively. In subsection 2.2, we have found that the expressions $\Phi_{o N^{\prime}}^{m}$ and $\Psi_{o N^{\prime}}^{n}$ are represented respectively by Eqs. (25) and (26) for $z=R / r$ approaching unity, i.e. $d$ approaching zero. Therefore, the expressions $\Phi_{D^{\prime}}^{m}$ and $\Psi_{N_{N}}^{m}$ can be adopted for the solutions of the diffusion equation for the exterior and interior problems, respectively.

\subsection{Numerical calculation}

The expressions (17), (19) and (18), (20) represent solutions of the diffusion equation for the exterior and interior problems of a torus with a ring source at $z=$ $z_{s}$, respectively.

In practice, a numerical evaluation of the associated Legendre functions can be performed by referring to standard works ${ }^{(\gamma)-(9)}$. We can evaluate $K_{p}^{1}$ and $K_{p}^{2}$ from Eqs. (22) and (24), respectively, which are expressed as series of the associated Legendre functions, and $S_{L}^{M}$ and $T_{L}^{M}$ from Eqs. (21) and (23), respectively, which are expressed as series of $K_{p}^{1}$ and $K_{p}^{2}$. Then by substituting the $S_{L}^{M}$ and $T_{L}^{M}$ into Eqs. $(17) \sim(20)$, we can obtain the solution of the neutron diffusion Eq. (3). We programmed a numerical calculating Fortran code with a truncation error of less than $10^{-6}$ for the solutions, by following above-mentioned procedure. The solutions of the diffusion equation in the toroidal coordinate were computed by utilizing

where 
Table 1. Examples of computations of $\phi_{e N}^{m}, \psi_{e N}^{m}, \phi_{O N^{\prime}}^{m}, \psi_{O^{\prime}}^{m}$

\begin{tabular}{|c|c|c|c|c|c|c|c|c|}
\hline \multirow{3}{*}{$\begin{array}{c}\psi \\
(\mathrm{deg})\end{array}$} & \multicolumn{4}{|c|}{$N=M+2 l, \quad M=|m|=2, \quad k d=4, \quad z=\cosh \sigma=1.25$} & \multicolumn{4}{|c|}{$N=M+2 l+1, \quad M=|m|=2, \quad k d=4, \quad z=\cosh \sigma=1.25$} \\
\hline & \multicolumn{2}{|c|}{$\phi_{e N}^{m}(z, \phi, \phi) e^{-i m \theta}$} & \multicolumn{2}{|c|}{$\psi_{* N}^{m}(z, \psi, \phi) e^{-i m \phi}$} & \multicolumn{2}{|c|}{$\phi_{O N^{\prime}}^{m_{m}}\left(z, \psi_{r} \phi\right) e^{-i m t}$} & \multicolumn{2}{|c|}{$\psi_{o w}^{n}(z, \phi, \phi) e^{-i m}$} \\
\hline & $l=0$ & $l=3$ & $l=0$ & $l=3$ & $l=0$ & $l=3$ & $l=0$ & $l=3$ \\
\hline 0 & $-2.667 E-5$ & $-3.154 E-4$ & $4.723 E+4$ & $3.679 E+3$ & 0 & 0 & $0^{\circ}$ & 1 \\
\hline 30 & $4.258 E-5$ & $-7.754 \quad E-4$ & 2. $444 E+3$ & $3.908 E+2$ & $1.939 E-4$ & $-5.768 E-3$ & 4. $346 E+3$ & $-1.447 E+1$ \\
\hline 60 & $3.541 E-4$ & $5.638 E-1$ & $6.821 E+1$ & $4.139 E+1$ & $2.190 E-3$ & $8.577 E-1$ & $1.365 E+2$ & $7.257 E+0$ \\
\hline 90 & 1.221E-3 & $2.166 E+1$ & $8.233 E+0$ & $3.948 E+0$ & $7.342 E-3$ & $8.342 E+1$ & $1.322 E+1$ & $7.483 E-1$ \\
\hline 120 & $2.380 E-3$ & $1.282 E+2$ & $2.519 E+0$ & $7.625 E-1$ & $1.121 E-2$ & $4.556 E+2$ & $2.707 E+0$ & $1.006 E-1$ \\
\hline 150 & 3. $321 E-3$ & $3.176 E+2$ & $1.367 E+0$ & $2.975 E-1$ & $8.576 E-3$ & $6.628 E+2$ & $7.286 E-1$ & $1.958 E-2$ \\
\hline 180 & $3.676 E-3$ & $4.213 E+2$ & $1.131 E+0$ & 2. $193 E-1$ & 0 & 0 & 0 & 0 \\
\hline
\end{tabular}

the Fortran code and tabulated in Table 1 as an example of numerical results.

\section{The Neutron Diffuion Phenomena from a Ring Source}

As an example of applications of the solutions expressed in Eqs. (17) (24), we consider the distribution of the flux $\Psi$ in an infinite homogeneous medium for an axisymmetric-toroidal ring source. The monoenergetic neutron diffusion equation is given by

$$
\left.\begin{array}{l}
\nabla^{2} \Psi-k^{2} \Psi=0 \\
k^{2}=\Sigma_{a} / D
\end{array}\right\}
$$

where $\Psi$ is the neutron flux, and $D$ and $\Sigma_{a}$ are respectively the diffusion coefficient and absorption cross section which are assumed to be constant in a medium. In this case, the solution of Eq. (37) is represented by $\mathrm{Eq}$. (17) with $m=0$ which is even function for $\psi$, as follows :

$$
\Psi(\sigma, \psi)=\sum_{i=0}^{\infty} a_{i} \phi_{e, 2 \ell}^{0}(\sigma, \psi),
$$

where $a_{\imath}$ are arbitrary constants. In order to determine the coefficients $a_{l}$, we impose the boundary condition $\Psi\left(\sigma_{s}, \psi\right)=\Psi_{0}$ on Eq. (38) and expand both the sides as Fourier consine series, so that we obtain simultaneous algebraic quations with infinite unknowns $a_{\iota}$,

$$
\delta_{n, 0}=\sum_{i=0}^{\infty} a_{l} U_{n, l},
$$

where $n, l$ are positive integer, and

$$
\begin{aligned}
\delta_{n, 0}= & \left\{\begin{array}{lll}
\Psi_{0} & \text { for } & n=0 \\
0 & \text { for } & n \neq 0
\end{array}\right. \\
U_{n, t}= & \frac{1}{2 \pi} \int_{0}^{2 \pi} \Phi_{e, 2 l}^{0}\left(\sigma_{s}, \psi\right) \cos n \psi d \phi \\
= & H_{n,-N-1}-H_{n, N}, \\
H_{n, L}= & \frac{\pi^{1 / 2}(k d)^{L}}{2^{L / 2+1}} \\
& \times \sum_{p=0}^{\infty} \sum_{x=0}^{l} \frac{(k d)^{2 p}(-L / 2)_{q}}{2^{p} p ! q !} *
\end{aligned}
$$

$$
\begin{aligned}
I_{\nu}^{n}\left(z_{s}\right)= & \frac{1}{2 \pi} \int_{0}^{2 \pi} \frac{\cos n \phi}{\left(z_{s}-\cos \phi\right)^{2}} d \psi \\
I_{k}^{n}\left(z_{s}\right)= & \frac{\Gamma(k+n)}{\Gamma(k) \Gamma(n)} \\
& \times \frac{\left(z_{s}^{z}-1\right)^{-k+1 / 2}}{2^{k-1}\left(z_{s}+\sqrt{z_{s}^{2}-1}\right)^{m-k+1}} \\
& \times F(-k+1, n-k+1, n+1 \\
& \left.1 /\left(z_{s}+\sqrt{z_{s}^{2}-1}\right)\right) \\
I_{k}^{n}\left(z_{s}\right)= & \frac{(-1)^{n} \Gamma(-k+1)}{\Gamma(-k+n+1)}\left(z_{s}^{2}-1\right)^{-k / 2} \\
& \times P_{-k}^{n}\left(z_{s} / \sqrt{z_{s}^{2}-1}\right)
\end{aligned}
$$$$
(k<1)
$$

$$
\begin{aligned}
I_{k+1 / 2}^{n}\left(z_{s}\right)= & \frac{\Gamma(k-n+1 / 2)}{\Gamma(k+1 / 2)}\left(z_{s}^{2}-1\right)^{-k / 2-1 / 4} \\
& \times P_{k-1 / 2}^{n}\left(z_{s} / \sqrt{\left.z_{s}^{2}-1\right)}\right. \\
& \quad(k=0, \pm 1, \pm 2, \cdots)
\end{aligned}
$$

Expressions (43) $\sim(46)$ are derived from the integral representations of the associated Legendre functions.

For a given aspect ratio of the toroidal neutron source, the coefficients of Eq. (38) are determined from Eq. (39) and then the neutron flux $\Psi$ is obtained from Eq. (38). In practice, under the aspect ratio $\cosh \sigma_{s}$ $=R_{s} / r_{s}=2.5$, we calculate the distribution of the thermal flux in the heavy water $\left(k=0.0093 \mathrm{~cm}^{-1}\right)$ and beryllium $\left(k=0.0481 \mathrm{~cm}^{-1}\right)$ for $R_{s}=100 \mathrm{~cm}, r_{s}=40 \mathrm{~cm}$, $\Psi_{0}=10 \mathrm{~cm}^{-2} \mathrm{~s}^{-1}$ by the use of Eq. (38) truncated with the ten terms. Then, the boundary condition is approximated with the relative error $\left|\left\{\Psi\left(\sigma_{s}, \phi\right)-\Psi_{0}\right\} / \Psi_{0}\right|$ of less than $10^{-4}$. The computational results are shown in Fig. 2, where equiflux curves present the values of the neutron flux $\Psi \mathrm{cm}^{-2} \mathrm{~s}^{-1}$. 


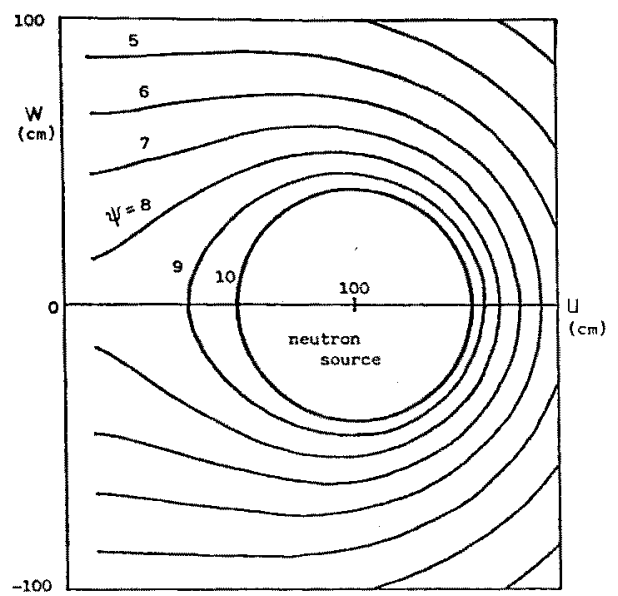

(a) $k=0.0093 \mathrm{~cm}^{-1}$ : heavy water

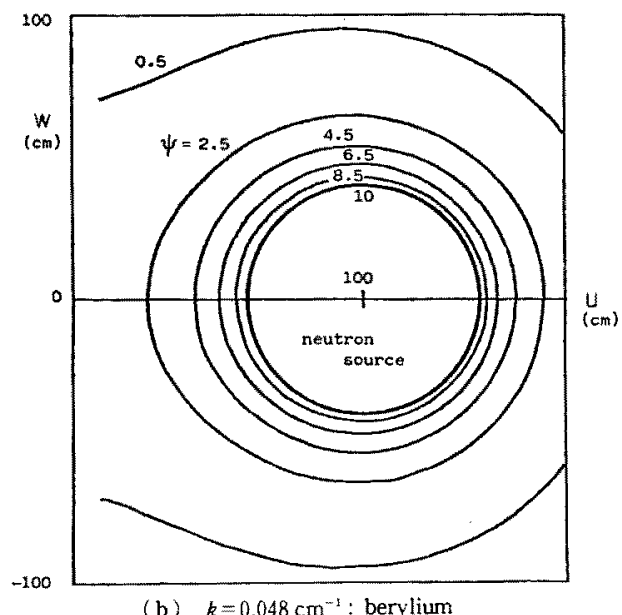

Fig. 2. Equiflux curves for $R_{s}=100 \mathrm{~cm}, r_{s}=40 \mathrm{~cm}$ and $\Psi_{0}=10 \mathrm{~cm}^{-2} \mathrm{~s}^{-1}$.

\section{Conclusion}

The neutron diffusion equation was analytically solved for the exterior and interior problems in toroidal coordinates and some computational results of the solution were shown in the table.

As an example of its applications, we evaluated the thermal neutron diffusion from a ring source in the heavy water and beryllium by expanding the boundary flux as a Fourier cosine series. The integrals in the Fourier coefficients are represented by the associated Legendre functions and Gauss' hypergeometric func* tions. The analytical solution of the neutron diffusion equation, in toroidal coordinates, may be useful for computational tool to investigate the toroidal effect of nutron diffusion in toroidal system.

The problem with the toroidal boundary condition can be solved under the approximation of an expasion with respect to the inverse aspect ratio in cylindrical coordinate, for the case with a sufficiently large aspect ratio $R / r$, while the treatment of the case with a small aspect ratio is very difficult in the cylindrical coordinate. However, we have treated here the problem without using an expansion with respect to the inverse aspect ratio but by using the analytical solution of the diffusion equation in the toroidal coordinate, whose arguments are aspect ratio $R / r, \phi$ and $\phi$.

The authors would like to thank Dr. T. Honma of Hokkaido University for useful discussions. This work has been supported by Grant in Aid for Scientific Reseach administrated by Ministry of Education.

\section{References}

(1) G. C. Pomraning \& C. A. Stevens: Nucl. Sci. Eng. 55, 359 (1974)

(2) J. Jung: ibid., 60, 74 (1976)

(3) P. F. Zeweifel : Reactor Physics, p. 28 (1973) McGraw-Hill Kogakusha Ltd.

(4) V. H. Weston: Quart. Appl. Math.s 15, 420 (1957)

(5) V. H. Weston: ibid., 16, 237 (1958)

(6) M. Seki \& 1. Kaji : Memoirs of Hokkaido Automotive Junior College (in Japanese), 8, 51 (1980)

(7) M. Seki, M. Kito, I. Kaji \& T. Honma: Bulletion of the Faculty of Engineering, Hokkaido University (in Japanese). $82,23(1976)$

(8) E. W. Hobson: The Theory of Spherical and Ellipsoidal Harmonics, p. 178, 433 (1965) Chelsa Publishing Company. New York 2nd Reprint

(9) P. M. Morse \& H. Feshbach: Method of Theoretical Physics, p. 1301 (1953) McGraw-Hill Book Company 